\title{
CARACTERIZANDO LA RESOLUCIÓN DE PROBLEMAS DESDE LA VARIACIÓN Y EL CAMBIO EN DOMINIOS DISCRETOS Y LA TEORÍA FUNDAMENTADA
}

\author{
CHARACTERIZING PROBLEM \\ SOLVING FROM VARIATION AND \\ CHANGE IN DISCRETE DOMAINS \\ AND GROUNDED THEORY
}

Luis Fernando Mariño',

Rosa Virginia Hernández ${ }^{2}$

\section{RESUMEN}

El estudio tuvo como propósito dar respuesta a la pregunta de investigación, ¿Cómo es la naturaleza de la resolución de problemas que involucran ecuaciones lineales diofánticas de la forma $a x+b y=c$ manifestadoporprofesores

1 Doctor en Educación Matemática (Universidad Antonio Nariño, Colombia). Magister en Educación Matemática. Licenciado en Matemáticas y Computación. Profesor Departamento de Matemáticas y Estadística de la Universidad Francisco de Paula Santander (Cúcuta). Correo. fernandoml@ufps.edu.co. Orcid: https://orcid. org/0000-0002-3438-6963

2 Magister en Educación Matemática. Licenciada en Matemáticas y Computación. Profesora adscrita al Departamento de Matemáticas y Estadística de la Universidad Francisco de Paula Santander (Cúcuta). Correo. rosavirginia@ufps.edu. Orcid: /orcid.org/0000-0002-2638-671X de matemáticas en formación? El trabajo estuvo orientado por un enfoque cualitativo con un diseño desde la teoría fundamentada. Como fuentes de datos se diseñaron e implementaron 6 actividades didácticas y una entrevista retrospectiva no estructurada a un grupo de 15 estudiantes que se forman para ser profesores de matemáticas. Entre los hallazgos se destacan las acciones variacionales manifestadas por los participantes, que les posibilitó ir de sustituciones y combinaciones variacionales a establecer relaciones para formalizar, generalizar, probar, 
organizar y reorganizar su conocimiento. El método de comparación constante, junto a los procesos de codificación (abierta, axial y selectiva), el muestreo y saturación teórica se constituyeron en la vía para interpretar y dar sentido a los datos posibilitando caracterizar la resolución de problemas en dominios discretos como un flujo permanente de acciones e interacciones entre procesos y subprocesos cuando los participantes resuelven problemas que involucran ecuaciones lineales diofánticas de la forma $a x+b y=c$

\section{PALABRAS CLAVE:}

Resolución de problemas, Teoría Fundamentada, Ecuaciones lineales Diofánticas, Variación y Cambio, Dominios Discretos.

\section{ABSTRACT}

The had as purpose answering the research question: What is the nature of problem solving involving linear diophantic equations of the form $a x+b y=c$ as expressed by mathematics teachers in training? The project was guided by a qualitative approach with a grounded theory design. As data sources, 6 didactic activities and a retrospective unstructured interview with a group of 15 students who are pursuing a mathematics teaching bachelor's degree were designed and implemented. Among the findings, the variational actions expressed by the participants stud out, which enabled them to go from substitutions and variational combinations to establishing relationships in order to formalizing, generalizing, testing, organizing and reorganizing their knowledge. The constant comparison method, together with code processing (open, axial and selective), sampling and theoretical saturation, became the way to interpret and make sense of the data, making it possible to characterize problem solving in discrete domains as a permanent flow of actions and interactions between processes and sub-processes when the participants solve problems involving linear diophantic equations of the form $a x+b y=c$

KEYWORDS: Problem solving, Grounded Theory, Diophantic linear equations, Variation and Change, Discrete Domains.

\section{INTRODUCCIÓN}

Todo ser humano se enfrenta diariamente a situaciones contradictorias, caracterizadas por obstáculos y dificultades, donde no se vislumbra fácilmente el camino o las vías de solución para el logro de algún objetivo. El término problema tiene diversas definiciones, desde un punto conceptual, se entiende por problema una dificultad teórica o práctica que provoca una actitud indagadora de una persona y que enriquece sus conocimientos (Kupisiewicz, 1964).

Para a Mayer (2010) un problema consiste en un estado dado, donde se describe la situación actual y un estado objetivo, es decir el estado o situación deseada junto a un conjunto de operadores o reglas para ir de un estado al otro. Por tanto, un problema se produce cuando una situación está en un estado, el solucionador quiere llevarla a otro estado, pero hay una serie de obstáculos que no permite que la transición entre estados fluya con facilidad.

Entre tanto, la resolución del problema se produce cuando el solucionador del problema se compromete con una actividad cognitiva dirigida a superar el problema (Mayer, 2010). Para Polya (1981) la resolución de problemas es como encontrar una salida a una dificultad, una forma de sortear un obstáculo. Según Gagne (1965) la resolución de problemas es el proceso mediante el cual la situación incierta es clarificada, e implica en mayor o menor medida, la aplicación de conocimientos y procedimientos por parte del solucionador. Mayer (2010) por su parte, define la resolución de problemas como un resumen de los procesos cognitvos centrados en el cambio del estado dado, al estado final donde el procedimiento de solución no es obvio. 
En el ámbito escolar uno de los grandes objetivos de la matemática es orientar al estudiante con situaciones y actividades que posibiliten potenciar y desarrollar su pensamiento matemático. Una posible vía para el desarrollo del pensamiento matemático es la resolución de problemas, pero en el aula de clase parece ocurrir todo lo contrario. Por un lado, las estrategias didácticas parecen estar más enfocadas al reconocimiento de fórmulas y procesos algorítmicos, por parte de los estudiantes. Además, los profesores se encargan de presentar los contenidos como un saber acabado y pulido de conocimientos, reconocidos por una comunidad matemática o de educación matemática, pero ajenos al estudiante (Dreyfus, 2002).

La resolución de problemas ha hecho aportes significativos tanto en la enseñanza como en el aprendizaje de la matemática y la investigación en educación matemática. Desde la heurística el más importante ha sido George Polya con su reconocida obra How to Solve it, Polya (1945) sugirió cuatro fases como marco para la resolución de problemas: entender el problema, diseñar un plan, llevar a cabo el plan y mirar hacia atrás en el trabajo.

Por su parte Schoenfeld (2016) caracterizó cinco dimensiones que intervienen directa, dinámica e inter-relacionadamente: 1) Dimensión cognitiva, base de conocimientos, 2) Heurísticas, estrategias en la resolución de problemas, 3) Dimensión meta cognitiva, monitoreo y control (autoregulación), 4) Dimensión afectiva, creencias y afectos y 5) Práctica matemática. Entre tanto, Mason, Burton y Stacey (2010), propusieron tres fases en la resolución de problemas: entrada, ataque y revisión. La fase de entrada abarca las dos primeras etapas de Polya mientras que el ataque y la revisión corresponden a la tercera y cuarta etapa de Polya (1945). En este mismo sentido Mayer (2010), divide la resolución de problemas en dos grandes fases, que las denominó representación del problema y solución del problema.
Desde otra mirada, la mayoría de investigaciones que describen y aportan en los procesos y estrategias en la resolución de problemas provienen de una diversidad de situaciones problemas en dominios continuos. Estas caracterizaciones generalmente se han realizado desde la óptica del investigador y no se han abordado, desde las acciones que evidencian el pensamiento matemático que puso en juego el solucionador para resolver el problema.

Por otra parte, y como lo afirmaron Stanic y Kilpatrick (1989) los problemas siempre han tenido privilegio en la matemática escolar, pero la resolución de problemas no. Sólo a partir de los años ochenta del siglo XIX, se asume la resolución de problemas como uno de los ejes en el currículo de matemáticas (Schoenfeld, 2016).

Las ecuaciones lineales diofánticas de la forma $a x+b y=c$ por su parte, se caracterizan porque tanto los números $a, b, c$ como las soluciones $x$ e $y$ pertenecen al grupo de los números enteros. Solucionar estas ecuaciones simplemente a una serie de procedimientos algorítmicos. En contraste, y aunque parezca sencillo resolverlas en dominios discretos (números enteros), requiere por parte del solucionador poner en juego una serie de operaciones de su pensamiento matemático, además de ingenio y creatividad.

Ante esta situación la investigación tuvo como propósito dar respuesta a la pregunta: ¿Cómo es la naturaleza de la resolución de problemas que involucran ecuaciones lineales diofánticas de la forma $a x+b y=c$, manifestado por profesores de matemáticas en formación?

\section{REVISIÓN DE LA LITERATURA}

La resolución de problemas ha sido investigada desde diversas fuentes, contextos y diferentes perspectivas. En lo que si parecen coincidir la mayoría de investigadores, es en dos elementos 
primordiales que se destacan en estos estudios. Por un lado, estan los procesos mentales o cognitivos que el solucionador pone en juego para resolver problema y por el otro, las estrategias para el resolver problema.

Polya (1945) en su reconocida obra How To Solve It, propuso cuatro fases para la resolución de problemas. La primera fase consiste en entender el problema, el resolutor debe tener claridad en ¿qué es lo requiere?, tiene que identificar las partes o elementos del problema, lo que se desconoce, los datos y las condiciones. Para identificar estos elementos se pueden hacer preguntas: ¿qué es lo desconocido?, ¿qué datos se conoce?, ¿cuáles son las condiciones?, incluso el solucionador puede preguntarse: ¿es posible resolver el problema, con estas condiciones?, ¿puede hacer una figura que represente el problema?, además, en esta fase se tiene que empezar a asignar nombres a los objetos, introduciendo la notación adecuada.

En la segunda fase, el solucionador tiene que intentar ver o descubrir la forma como se relacionan y se conectan los diferentes elementos del problema y ¿cómo lo desconocido se relaciona con los datos?, para tener una idea de la solución y diseñar un plan. En la tercera fase se ejecuta el plan teniendo en mente la idea de la solución, y en la cuarta fase se mira la solución completa, se revisa y se discute sobre ella.

Schoenfeld (2016), además de las cinco dimensiones con la que caracterizó la resolución de problemas, propuso acciones para la enseñanza: antes, durante y después en la resolución de problemas. Antes de la resolución, discutir palabras o frases que los estudiantes no entiendan, usar la discusión con toda la clase para enfocarse en la importancia de entender el problema y discutir en clase las posibles estrategias para resolver un problema.

Durante la resolución, observar y preguntar a los alumnos para determinar dónde y cómo están, proporcionar sugerencias si es el caso, proponer extensiones del problema si es necesario y pedir a los estudiantes que obtengan una solución que responda a la pregunta.

Después de la resolución, socializar y discutir soluciones, establecer relaciones con problemas previamente resueltos o hacer que los estudiantes resuelvan problemas como extensiones del mismo y discutir las características especiales, por ejemplo, imágenes, tablas, etc.

Mason, Burton y Stacey (2010) propusieron tres fases en la resolución de problemas: entrada, ataque y revisión. La fase de entrada abarca las dos primeras etapas de Polya mientras que el ataque y la revisión corresponden a la tercera y cuarta etapa de Polya (1945). En la fase de entrada, el potencial solucionador de problemas se familiariza con el contexto del problema. Conociendo el problema y jugando con las ideas, por ejemplo, a través de la particularización, especificando claramente lo que sabe, lo que desea, y considerando cuidadosamente lo que puede hacer.

Allí ocurre un cambio cualitativo, al atacar el problema usando las ideas exploradas en la fase anterior. Esta fase puede ser exitosa, pero a menudo puede conducir a un callejón sin salida aparente en que el individuo debe revisar lo que ha hecho hasta el momento y volver a la fase de entrada para considerar un nuevo ataque. Mason, Burton y Stacey (2010), afirman que una vez que algún tipo de solución se logra, el estado de ánimo cambia nuevamente a una revisión más elaborada (comprobar los resultados para asegurarse de que no se ha cometido ningún error). Comprende aprender de estrategias que fueron útiles en otras ocasiones y luego estar preparado para ampliar o llevar el problema a otros niveles de sofisticación, volver a iniciar el ciclo de entrada a un nivel más sofisticado.

Para Mayer (2010), la resolución del problema puede dividirse en dos grandes fases: repre- 
sentación del problema y solución del problema. La fase de representación implica la representación mental del problema, esto incluye el proceso cognitivo de representar. Representar en el sentido de construir un modelo de la situación, es decir un modelo mental de la situación que describe el problema.

Mientras que la fase de solución del problema implica llevar a cabo un plan para resolver el problema. Esto incluye los procesos cognitivos de planificación (elaborar un plan), ejecutar (lleva a cabo el plan) y supervisar, es decir el seguimiento a la eficacia del plan. Mayer (2010) afirma también, que para resolver el problema con éxito son necesarios varios tipos de conocimiento: hechos (conocer el mundo), conceptos, procedimientos, estrategias y creencias.

\section{METODOLOGÍA}

La pregunta de investigación acerca de ¿cómo es la naturaleza de la resolución de problemas manifestado por los participantes?, en el contexto propuesto, conduce necesariamente a los cuestionamientos: ¿cuáles son las propiedades y dimensiones de la resolución de problemas? (lo ontológico), ¿cómo es?, ¿cómo se desarrolla?, (lo epistemológico). Por tanto, para intentar responder estas cuestiones se optó por el enfoque cualitativo con un diseño desde la teoría fundamenta siguiendo a Glasser y Strauss $(1967,1978,2017)$, que condujo el estudio a interpretar, dar sentido y describir los datos manifestados por los participantes cuando su pensamiento opera sobre este tipo de problemas.

Ante estos cuestionamientos, la primera acción dada, consistió en hacer claridad entre lo que son las operaciones del pensamiento de los participantes cuando resuelven problemas que involucran ecuaciones lineales diofánticas de la forma $a x+b y=c$ y los resultados de estas operaciones, manifestadas por expresiones verbales y escritas de los participantes, que a su vez se convirtieron en los datos sobre los cuales se construyó la teoría.

\subsection{Los participantes}

El estudio se realizó con 15 alumnos que tomaron un curso de Teoría de Números y se forman para ser profesores de matemáticas en la Universidad Francisco de Paula Santander, institución de carácter público en la ciudad de Cúcuta, durante el I semestre del año 2020. La selección de los participantes obedece al propósito del estudio, de caracterizar la resolución de problemas desde dominios discretos (el contenido del curso involucra la temática de ecuaciones diofánticas), en contraste a los contextos en dominios continuos, donde tradicionalmente ha sido caracterizada la resolución de problemas. Las edades de los participantes oscilaban entre 18 y 23 años, cerca de la tercera parte de ellas son mujeres.

\subsection{El diseño de investigación desde la teo- ría fundamentada}

Siguiendo a Corbin y Strauss (1990, 2008, 2017) y Charmaz $(2006,2014)$, cuando las investigaciones se abordan con un enfoque desde la teoría fundamentada deben caracterizarse por abordar al menos, los siguientes elementos: a) trabajo simúltaneo entre la recolceción y análisis de datos, b) la construcción de códigos y categorías a partir de los datos, no hipótesis preconcebidas, c) avanzar en el desarrollo de la teoría durante cada paso de recolección y análisis de datos, d) el muestreo se dirige a la construcción de la teoría, no a la representatividad de una población determinada y e) el método de comparación constante, en la búsqueda de similitudes, diferencias y relaciones entre las categorías construidas.

\subsection{Fuentes de datos}

Se diseñaron, rediseñaron e implementaron 6 actividades didácticas y una entrevista retrospectiva no estructura como adaptación de la 
entrevista clínica de Piaget. Las actividades didácticas involucran problemas relacionados con ecuaciones lineales diofánticas de la forma $a x+b y=c$, como fuentes de datos.

Las actividades didácticas se caracterizaron por los siguientes elementos que conformaron su estructura: 1) Encabezamiento, conformado nombre institución, fecha, tiempo de la actividad, indicaciones, etc.; 2) Presentación, basada en temas específicos relacionados con la historia de la matemática; 3) Desarrollo de la actividad, se inicia siempre con el planteamiento de un problema, para que cada participante lo resuelva individualmente o intente resolverlo con los conocimientos matemáticos que tiene a su disposición, las demás tareas de cada actividad, la conforman una serie de cuestiones o preguntas diseñadas con la intención de orientar a los participantes para que sean ellos mismos, quienes elaboren y construyan su conocimiento desde la variación y el cambio; y 4) Evaluación, conformada por una serie de problemas retadores.

\subsection{La estrategia puesta en marcha}

La Figura 1, esquematiza la estrategia puesta en marcha con un diseño desde la teoría fundamentada. Se organizaron tres intervenciones con los participantes, cada una de ellas agrupó dos actividades didácticas de acuerdo a las características de los problemas involucrados. Luego de la primera intervención fueron seleccionados los primeros incidentes y se empieza el primer ciclo de codificación abierta y axial que dio origen a los primeros códigos, luego de este análisis y proceso de codificación, se realizan algunas modificaciones a las actividades del grupo 2. Se procedió a la segunda y tercera intervención y a los procesos paralelos de análisis y codificación que finalmente condujeron a la categoría central como núcleo de la teoría.

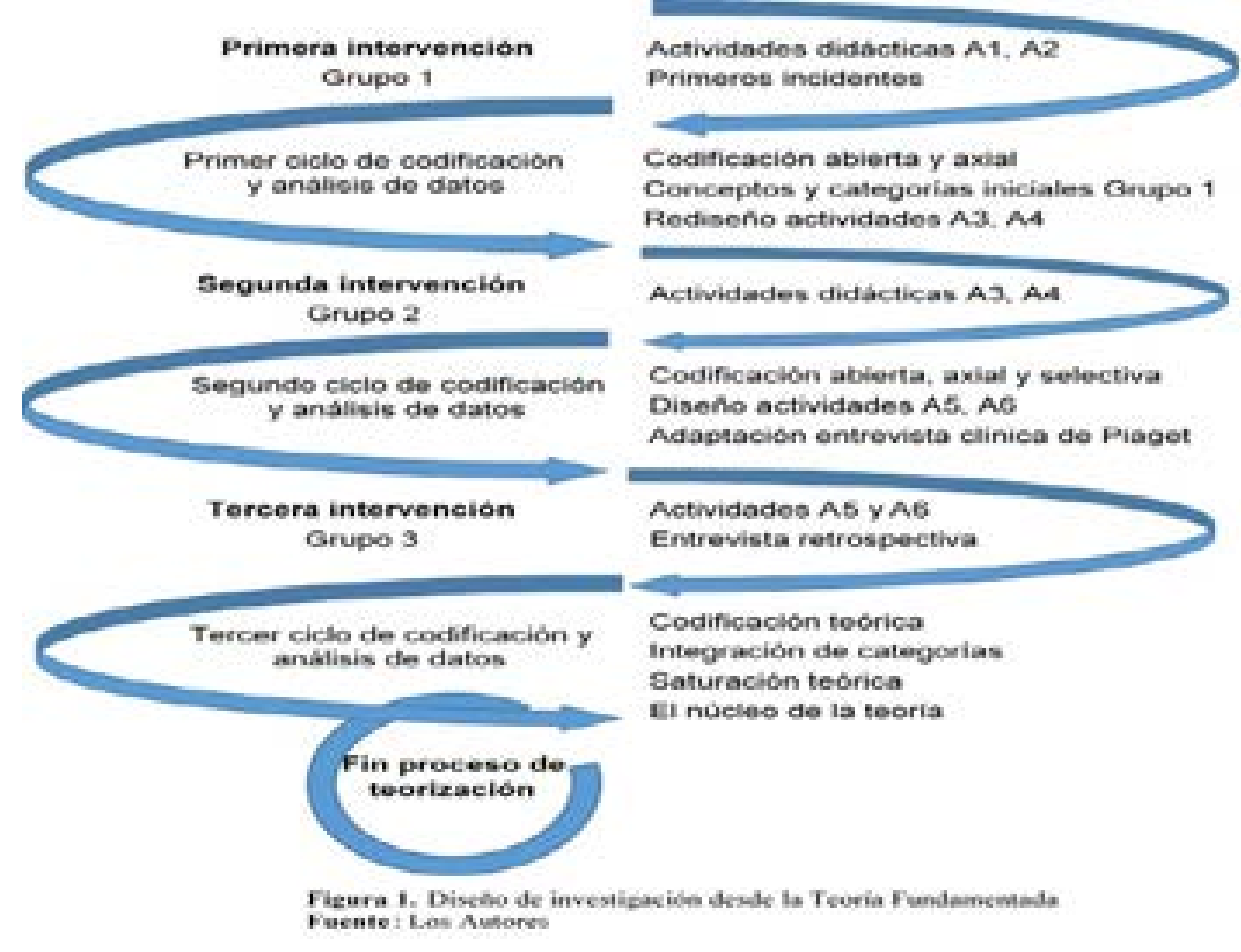

Figura 1. Diseño de investigación desde la Teoría Fundamenta.

Fuente: Autores 
4. LOS PROCESOS SIMULTÁNEOS DE RECOLECCIÓN, ANÁLISIS DE DATOS, CONSTRUCCIÓN Y EVOLUCIÓN DE LA TEORÍA

Siguiendo el diseño de investigación propuesto a continuación, se muestra la manera como fueron emergiendo las categorías de los datos, se fue elaborando y construyendo la teoría, hasta llegar a la categoría central o núcleo de la teoría formal, a partir de los procesos simultáneos de recolección, codificación (abierta, axial, selectiva) y análisis de datos.

\subsection{Primera intervención, primer ciclo de codificación y análisis de datos}

En la primera intervención, los participantes trabajan las actividades $A 1$, que involucra problemas relacionados con el máximo común divisor de los números $a$ y $b$ implicados en la solución a la ecuación $a x+b y=c$, así como la representación del, $\operatorname{mcd}(a, b)=a s+b t$ con la intención de inducir al estudiante en procesos de solución a esta ecuación desde la var- iación y el cambio. La actividad A2 por su parte, hace referencia a la solución a la ecuación lineal diofántica de la forma $a x+b y=c \quad$ utilizando el método de la pendiente.

El primer problema a resolver en la actividad A2, consistió en que los participantes resolvieran la ecuación lineal diofántica$$
6 x+21 y=102
$$

La primera acción de los participantes fue trabajar en el dominio de números reales, pero cuando se aclaró que el problema debía solucionarse en el contexto de los números enteros, el problema no fue nada fácil.

Por tanto, el profesor pide a los estudiantes que trabajen sobre la Cuestión 1. La Figura 1, muestra los resultados de la estudiante codificada como E6. Los trabajos de esta estudiante se codificaron como E6 A2 EDLI, donde E6 es el código del participante, $\mathrm{A} 2$ el número de la actividad y EDLI hace referencia al tema ecuaciones diofánticas lineales I. De esta manera se codificó el trabajo de todos los estudiantes.

\begin{abstract}
Cuestión 1. Dada la ecuación $6 x+21 y=102$ con coeficientes enteros. Encuentre varias parejas de námeros enteros que se les puedan asignar a las variables $x$ e $y$, do tal manera que la igualdad se cumpla. Eecriba los posibles valores para estas variables en la siguiente tabla. La tercera columna es para que verifique la veracidad de la iguaidad
\end{abstract}

\begin{tabular}{|c|c|c|}
\hline$x$ & $y$ & $6 x+21 y=\imath \ldots ?$ \\
\hline 3 & 4 & $6(3)+21(4)=102$ \\
\hline$A D$ & 2 & $6(1)+21(2)=102$ \\
\hline 17 & 0 & $6(07)+21|0\rangle=407$ \\
\hline 2,4 & -2 & $6(24)+21(-2)=\operatorname{ton}$ \\
\hline 21 & -4 & $6(30)+21(5 y)=102$ \\
\hline 38 & -6 & $6(3 x)+21(-6) \times 10 ?$ \\
\hline
\end{tabular}

Figura 2, Sustitución y combinacién variacional E6 A.2 EDLI Fueate: Los Autores

Figura 2. Sustitución y combinación Variacional E6 A2 EDLI

Fuente: Autores 
En la Cuestión 2, se le propuso a los participantes: Analice la columna de los valores para ¿Encuentra algún patrón o relación entre estos valores?, escríbalos. Hago lo mismo con los valores de la variable. Ante esta cuestión la estudiante E6, responde:

E6: En la columna de las $\mathrm{x}$ tiene una relación de más 7 es decir si le damos el valor a $x=3$ el siguiente valor $x=10$

En la columna de las entre más pequeño sea el valor de la este va a ser mayor entre más grande sea el valor de " $x ", " y "$ es menos y va aumentando de dos en dos.

Ante la cuestión 3: ¿Qué relación o que condición considera usted que deben cumplirse para que se cumpla la igualdad?, explique. La participante E6, responde:

E6: Debe existir un divisor que divida a los valores $a, b y c$

Análisis de las acciones manifestadas por E6. En las acciones como respuesta a la Cuestión 1 , se observa como ella empieza a sustituir números en los valores de $x$ e $y$ que hacen verdadera la ecuación.

En lo que respecta a la Cuestión 2, empieza a establecer nexos y relaciones entre cada una de las variables $x$ e $y$ de forma independiente, pero a la vez establece relaciones entre la variación conjunta de las dos variables para que la igualdad se cumpla. Mientras que en las repuesta a la Cuestión 3, establece una condición necesaria para que la ecuación tenga solución en los números enteros.

La Figura 3, muestra el proceso realizado por el participante E15, ante la Cuestión 1:

\begin{tabular}{|c|c|c|}
\hline$x$ & $y$ & $6 x+21 y=z \ldots ?$ \\
\hline-11 & 8 & $6(-19)+21(8)=102$ \\
\hline-4 & 6 & $6(-4)+6(6)=102$ \\
\hline 3 & 4 & $6(3)+6(4)=102$ \\
\hline 10 & 2 & $6(10)+6(2)=102$ \\
\hline 17 & 0 & $6(11)+6(0)=102$ \\
\hline 24 & -2 & $6(24)+6(-2)=102$ \\
\hline
\end{tabular}
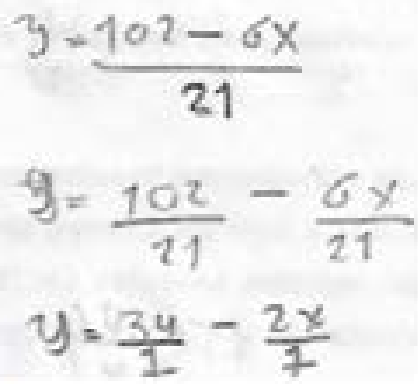

Figura 3. Sustituciones y combinaciones E6 A.2 EDL.I

Fuente: Los Autores

Figura 3. Sustituciones y combinaciones E6 A2 EDLI

Fuente: Autores

Lo primero fue, hacer uso de procedimientos algebraicos despejando la variable $y$ :

E15:

$$
y=\frac{(102-6 x)}{21}
$$

$$
y=\frac{34}{7}-\frac{2}{7} x
$$

luego simplifica
A la Cuestión 2, responde:

E15: pues mirando la pendiente de la ecuación podemos ver que la diferencia entre cada valor de es de 7 y entre cada valor de "y" la diferencia es de 2.

Mientras que a la cuestión 3, responde: 
disminuyen proporcionalmente en base a un valor que depende de la pendiente.

Análisis de las acciones manifestadas por E6: para responder la cuestión 1 E15 opta por un tratamiento a la ecuación como función, como estrategia para hallar números para las variables e de tal manera que hagan verdadera la ecuación, como se observa en la Figura 3. En las respuestas dadas a las cuestiones 2 y 3 encuentra patrones acerca de cómo varían los valores de $x$ e $y$ de manera independiente, pero simultáneamente establece una condición conjunta de las dos variables que hacen verdadera la ecuación y dependen proporcionalmente de la pendiente.

\subsection{Construyendo la teoría, los primeros có- digos y categorías}

Lo expresado en párrafos anteriores evidencia el proceso y la forma en que se interpretaron los resultados manifestados por los participantes, analizando palabras, frases, signos, símbolos, fórmulas, etc., dando sentido y describiendo los datos. Las actividades fueron analizadas una a una en su totalidad y no pregunta a pregunta, con el propósito de no perder el sentido de los datos.

Luego de este primer proceso de codificación abierta y axial emergen los primeros conceptos indicadores. Se denominan así, puesto que surgieron directamente de los datos y son indicadores de los datos. Los primeros conceptos indicadores o simplemente códigos como se les quiera llamaron se denominaron sustitución y combinación variacional. La Tabla 1, muestra estos conceptos junto a su descripción, propiedades y dimensiones.

Tabla 1. Códigos iniciales

\begin{tabular}{ll|l|l|}
\hline \multicolumn{1}{c|}{ Código } & \multicolumn{1}{c|}{ Propiedad } & \multicolumn{1}{c|}{ Dimensión } & \multicolumn{1}{c|}{ Descripción } \\
\hline $\begin{array}{l}\text { Sustitución var- } \\
\text { iacional }\end{array}$ & $\begin{array}{l}\text { Asignar números enteros } \\
\text { a las variables que hagan } \\
\text { verdadera la ecuación }\end{array}$ & $\begin{array}{l}\text { Tipos de números } \\
\text { enteros asigna- } \\
\text { dos }\end{array}$ & $\begin{array}{l}\text { Reemplazar las vari- } \\
\text { ables por números en- } \\
\text { teros positivos o nega- } \\
\text { tivos. }\end{array}$ \\
\hline \multicolumn{2}{|l|}{ Lo que varía: Números enteros positivos o negativos signados } \\
\hline
\end{tabular}

El proceso de análisis y codificación sigue su curso. Las tablas que muestran codificación axial presentan cambios sustanciales respecto a las tablas de codificación abierta puesto que, para trabajar las relaciones entre las categorías, Strauss y Corbin (1990) sugieren examinar los datos y los códigos basados en un paradigma de codificación que se centra y relaciona las condiciones causales, el contexto, las condiciones de intervención, las estrategias de acción/interacción y las consecuencias. La Tabla 2, muestra los inicios del proceso de codificación axial. 
Tabla 2. Inicio codificación axial

\begin{tabular}{cc|l|l}
\hline \multicolumn{1}{c}{ Codificación abierta } & \multicolumn{1}{c}{ Codificación axial } \\
\hline Nombre código & \multicolumn{1}{c}{ Nombre categoría } & \multicolumn{1}{c}{ Propiedades } & \multicolumn{1}{c}{ Dimensiones } \\
\hline Sustitución variacional & $\begin{array}{l}\text { Transformación var- } \\
\text { iacional }\end{array}$ & $\begin{array}{l}\text { Sustituir, combinar } \\
\text { parejas de números } \\
\text { enteros. }\end{array}$ & $\begin{array}{l}\text { Tipos de solución par- } \\
\text { ticular }\end{array}$ \\
\hline Combinación variacional
\end{tabular}

Descripción: Tipos de soluciones como resultado de las operaciones del pensamiento de sustituir y combinar variacionalmente.

Variable: Sustituir y combinar números enteros que cumplan las condiciones para las variables $x$ e $y$.

Cambio: Los diferentes soluciones a la ecuación diofántica lineal.

Contexto y condiciones de intervención: Actividad 2 presencial en el salón de clase y en línea después del inicio de cuarentena debido al Covid-19. Trabajo grupal e individual.

Estrategia: Actividad didáctica A2. Tema: Ecuaciones Lineales Diofánticas I

Objetivo de aprendizaje: Construir procedimientos para hallar soluciones en números enteros a ecuaciones lineales en dos variables con coeficientes enteros.

Estrategias de acción/interacción: Los estudiantes se proponen resolver problemas que involucran EDL utilizando diversas estrategias inductivas desde la variación y el cambio.

Consecuencias: cada estudiante puede generar diferentes estrategias para solucionar los problemas, así como diferentes formas de entender y de pensar el proceso.

Evidencia: Material impreso y digital.

\subsection{Segunda intervención, segundo ciclo de codificación y análisis de datos}

En la actividad $\mathrm{A} 4$, se propone el siguiente problema retador a los participantes: Dada la ecuación lineal diofántica $a x+b y=c$ números $\mathrm{a}, \mathrm{b}$ y $\mathrm{c}$, para que la ecuación tenga solución únicamente en los enteros positivos?. La Figura 4, muestra las acciones realizadas por el estudiante E15.

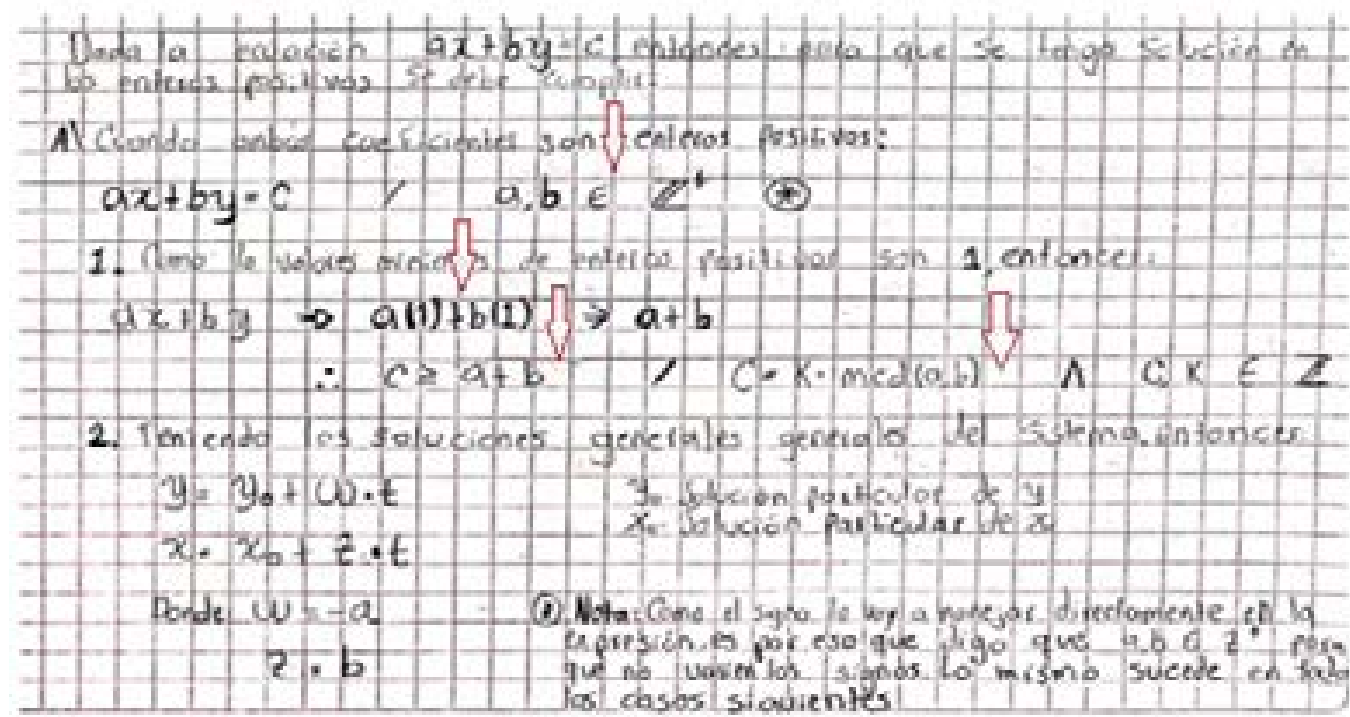

Figura 4. Generalización desde la variación y el cambio E15 A4 EDL II Fuente: Los Autores 
Análisis de las acciones manifestadas por E15. A estas alturas del desarrollo del curso, las acciones del estudiante E15 muestran un trabajo mejor elaborado, que al inicio del mismo. Ante el problema, la primera acción consiste en establecer una relación entre los números $x$ e $y$ como soluciones y los números a y $b$. Como los números tienen que ser enteros positivos, E15 establece que los números a y $b$ deben pertenecer a los enteros positivos, como una condición necesaria y suficiente. Como el estu-

$$
\text { diante lo afirma } a, b \in \mathrm{Z}^{+}
$$

Se evidencian allí, las operaciones de sustitución y combinación variacional, construidas en el primer ciclo de análisis, puesto que sustituye los valores de $x$ e $y$ por el número, puesto que es el primer entero positivo. Inmediatamente establece otra relación, que $c \geq a+b$. Se observa también, como el estudiante asigna letras, signos y fórmulas para representar y organizar y estructurar su conocimiento. De los procesos de análisis y codificación, tanto del trabajo de este participante y el de sus compañeros surgen las categorías, formalizar, formular, representar, organizar y reorganizar el conocimiento, entre otras.

\subsection{Tercera intervención, tercer ciclo de codificación y análisis, en la búsqueda de la densidad del núcleo de la teoría}

En el primer y segundo ciclos de codificación se integran la codificación abierta, axial y selectiva. Sin embargo, el análisis se centra más en la codificación abierta y axial; la codificación abierta puesto que, los conceptos y/o categorías iniciales surgen directamente de los datos, mientras que en la codificación axial el investigador se aleja un poco de los datos en la búsqueda de diferencias y similitudes para generar categorías y subcategorías.

Lo que se presenta a continuación es un proceso de codificación selectiva a un nivel más abstracto, trabajo que conduce a la integración de categorías y su densidad de relaciones. La integración al interior de las categorías y sus subcategorías se presenta mediante figuras como diagramas que esquematizan estas relaciones.

Los diagramas integradores son una forma visual de mostrar la integración acumulada de categorías, sus propiedades y relaciones. Muestran como las categorías y subcategorías se relacionan con la categoría central. Densidad en el sentido de conectarse no el sentido matemático de relaciones de inclusión, orden, dependencia, etc.

El esquema de la Figura 5, muestra una red de la densidad de relaciones en la categoría que en su momento se denominó estrategias variacionales en la resolución de problemas, luego del proceso de codificación selectiva. Es de vital importante aclarar que, en los procesos de codificación y análisis de datos, algunas categorías desaparecen o las absorben otras categorías de carácter más general, incluso varias de ellas se les cambia el nombre inicial asignado, atendiendo a las propiedades y dimensiones que emergen de los datos. 


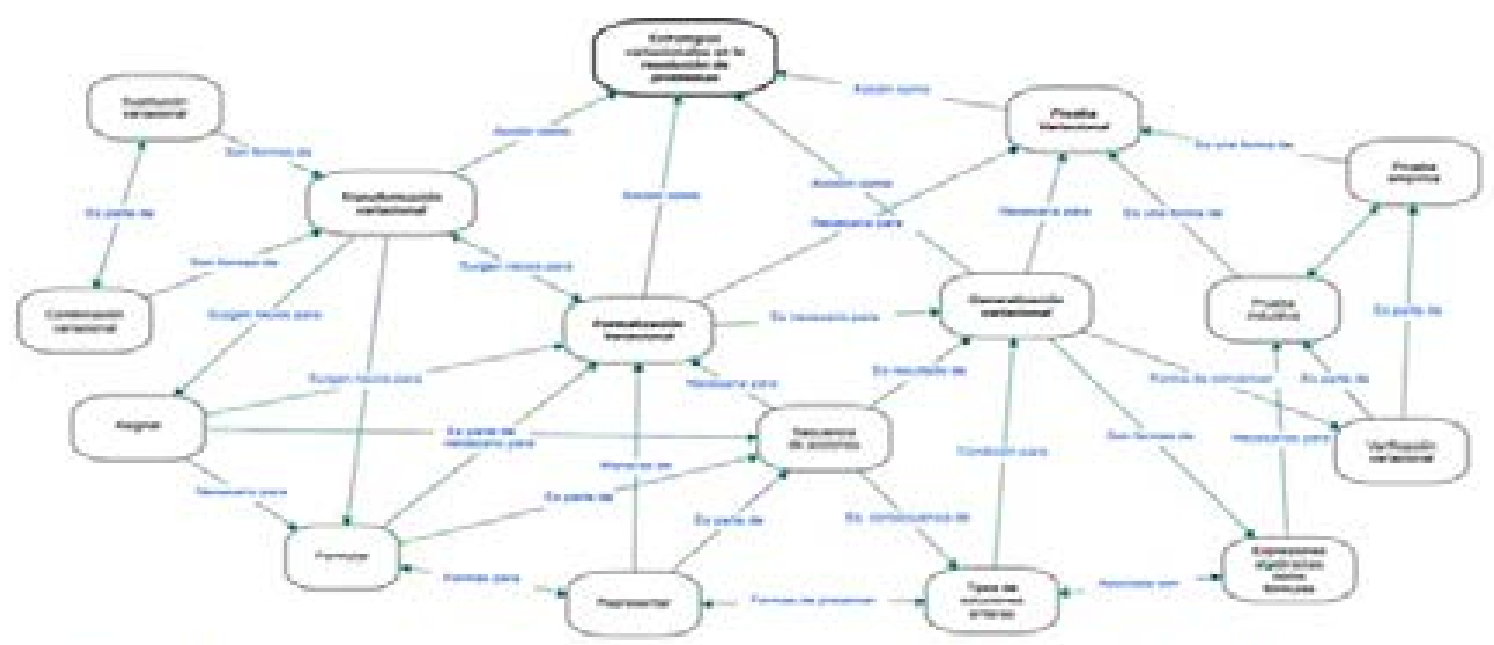

Figura 5. Densidad categoria estrategias variacionales en la resolucón de problemas Fuente: Los Autores

Figura 5. Densidad de relaciones categoría estrategias variacionales en la resolución de problemas

Fuente: Autores

\subsection{Muestreo teórico y saturación teórica}

Un proceso en evolución permanente. Primero se utiliza un muestreo por conveniencia para acceder a los participantes. Como resultado de la primera intervención, el primer ciclo de codificación y análisis de datos se utiliza el muestreo intencional, en la búsqueda de los primeros incidentes. Incidentes en el sentido que son mejores respuestas de los participantes de acuerdo al propósito del estudio. Se selecciona un grupo conformado por ocho resultados como formas de solución a los problemas propuestos en las primeras actividades, como incidentes.

Surgen de allí los primeros conceptos indicadores. Por ejemplo, el concepto transformación variacional. Además, se rediseñan las actividades. El proceso continúa con la segunda intervención y el segundo ciclo de análisis de datos. Se inicia el muestreo teórico, se aprende más sobre los datos dando profundidad a la teoría.

Se procede a la tercera intervención con nuevas vías de muestreo; el análisis se desarrolla a un nivel más abstracto. En la entrevista los partici- pantes responden preguntas relacionadas con las categorías construidas hasta ese momento. La intención era que descubrieran vínculos entre los elementos del problema, los explicaran, y generalizaran procesos y estrategias de solución. Esto permitió saturar las categorías o conceptos. Saturar en el sentido que los nuevos datos no aportan nuevas propiedades o dimensiones a las categorías desarrolladas y construidas.

Finalmente se logra la teoría formal con un alcance caracterizado por el muestreo teórico de relaciones, además de relaciones de similitud entre grupos y la diversidad de conceptos de los datos, como resultado del proceso de codificación y análisis siguiendo las condiciones y límites en el contexto propuesto.

De la transcripción, codificación y análisis de la entrevista y las soluciones escritas, se muestra el aporte de dos participantes en la saturación de categorías. PI significa profesor investigador y E10 el estudiante. La entrevista fue realizada el 09/06/2020 a las 9:30 am. La transcripción se hizo utilizando las mismas palabras del participante. La Cuestión 1, consistía: 
Plantee (invente) una ecuación dando valores a los números enteros de tal forma que tenga solución en los numeros enteros.

El estudiante propuso la ecuación lineal diofántica $7 x+15 y=343$

PI: ¿Cómo hizo o qué estrategia utilizó para construir la ecuación?

E10: Pues, la estrategia primeramente yo ya había hecho un progreso, entonces yo tengo ya planteada una fórmula en cual ya comprobé y es de manera significativa ya que por ella puedo establecer cualquier ecuación sin ningún inconveniente. Teniendo en cuenta de que el valor de $a y b$, es decir 7 siendo a y 15 siendo btienen un máximo común divisor y de esa forma pues con la fórmula que yo plantee no tengo que calcular el máximo común divisor, porque ya haciendo o encontrando el valor de ellos están relacionado con los valores numéricos de $a$ y $b$ de esa forma ya queda establecida no tengo inconveniente con la primera condición necesaria para las ecuaciones diofánticas.

PI: ¿Y cuál es esa condición?
E10: La condición es que $a$ y $b$ sean cualquier número arbitrario que pertenezca a los enteros positvos y el valor de es un producto en relación a ellos dos, donde yo aplico una fórmula que se menciona que es es igual a por $n$ mas bque multiplica a por $u$, siendo el número de soluciones que yo quiera colocar en la solución a plantear.

Comentarios y análisis. El participante habla de un progreso que ya había hecho, este progreso hace referencia al trabajo realizado en actividades anteriores, y lo muestra la Figura 6, como respuesta al problema: ¿qué condiciones debe imponerse a los números $a, b$ y $c$ para que la ecuación $a x+b y=c$ tenga únicamente cuatro soluciones en los enteros positivos?

Se evidencia allí la estrategia y habilidad del estudiante para sustituir y combinar números enteros y encontrar relaciones entre las diversas sustituciones y combinaciones que producen diferentes soluciones. Cada nueva sustitución y combinación transforma una solución en otra. En esta misma figura se observa la fórmula que el estudiante expresa en palabras y había construido con anterioridad, es decir $c=a n+b(a u)$ donde, $n<b$ y u=número de soluciones requeridas. 


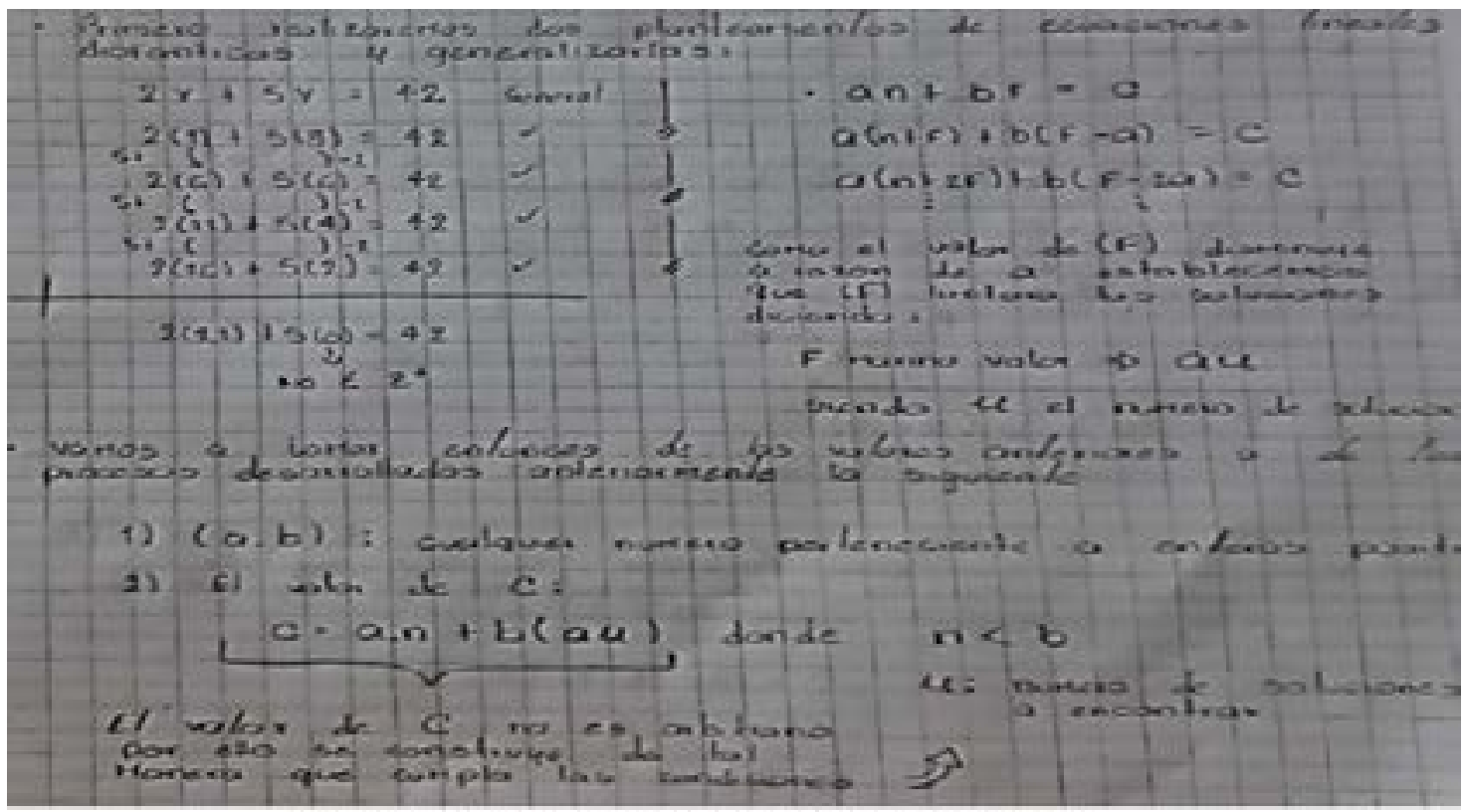

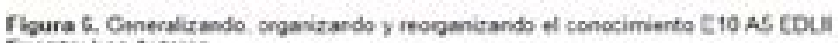

Feente: Loi Autsos:

A estas alturas del curso se puede afirmar que los participantes resuelven los problemas de una forma más elaborada. Por ejemplo, en la parte superior izquierda de la Figura 6, se evidencia como el estudiante E10, sustituye y combina números enteros y establece relaciones y condiciones. Utiliza esas relaciones y condiciones asignado, formulando y representado su conocimiento.

A otra pregunta, el participante a quien se le asignó el código $E 15$, respondió en la entrevista el día 11/06/2020 a las 3:00 pm:

PI: Si nosotros tenemos la ecuación lineal diofántica $a x+b y=c$ y queremos o exigimos que tenga un número determinado soluciones por ejemplo en los enteros positivos. ¿Explíquenos cómo se haría eso, o cómo respondió esta cuestión?

E15: Eso es para hallar un numero de $n$ soluciones al sistema, entonces lo primero que procedemos hacer es hallar las soluciones generales del sistema trabajando de forma general para primero avanzar y luego retroceder. Entonces tenemos una ecuación $a x+b y=c$ entonces por el método de pendiente y solución particular hallamos lo que son las soluciones generales, entonces $x$ es igual a la solución particular en $x$ más delta de $x, y$ a la solución particular en $y$ menos delta de $y$. Entonces como necesitamos hallar soluciones particulares en los positivos, entonces hacemos tanto a como a mayor a cero.

Comentario y análisis. Aunque no se evidencia de forma explícita, el participante explica cómo a partir de una solución particular de la ecuación y la pendiente de la función surgen nuevas soluciones de la forma $\left(x_{0}+\Delta x, y_{0}-\Delta y\right)$. Es decir, muestra una forma de sustituir y combinar utilizando la pendiente para transformar una solución conocida $\left(x_{0}, y_{0}\right)$, en una nueva solución a la ecuación.

El estudiante utiliza el término sistema para referirse a la ecuación $a x+b y=c$, hizo falta 
indagar en el participante el por qué el uso de este término.

Una mirada y análisis desde otro punto de vista, muestra que cada participante utiliza y combina estrategias que lo llevan de un estado a otro en los procesos para resolver las preguntas y problemas que se le plantearon.

Este proceso de muestreo por conveniencia, intencional y teórico junto a los procesos de codificación y el análisis de datos condujeron a una forma de saturar teóricamente la subcategoría de transformación variacional, desde diferentes fuentes de información.

\section{RESULTADOS: EL NÚCLEO DE LA TEO- RÍA EMERGENTE}

Como resultado del proceso de codificación, el método de comparación constante, el muestreo y saturación teórica, surge la categoría central como proceso que conforma el núcleo de la teoría, que se denominó operaciones variacionales en la resolución de problemas desde dominios discretos.

Siguiendo a Corbin y Strauss (2017) un proceso representa el ritmo, así como las formas cambiantes y repetitivas de acción-interacción, más las pausas e interrupciones que se producen cuando las personas actúan e interactúan con el propósito de alcanzar una meta o resolver un problema. El proceso tiene ciertas propiedades: a) es de naturaleza variable, b) hay diferentes formas de conceptualizar el proceso, c) tiene una rutina acción-interacción, y d) el proceso puede desglosarse en subprocesos. La Figura 7 , esquematiza el núcleo de la teoría o categoría como proceso en la resolución de problemas.

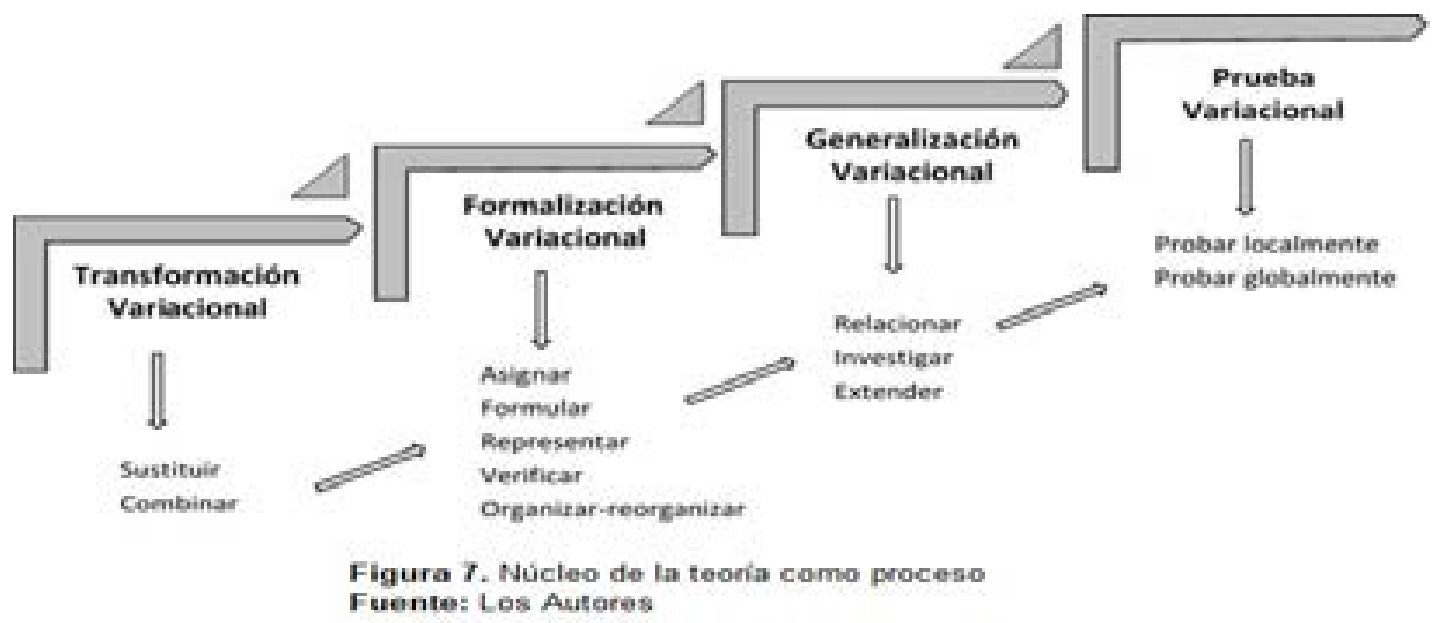

A continuación, se definen estos procesos y subprocesos desde la variación y el cambio que conformaron el núcleo de la teoría y que se denominó, operaciones variacionales en la resolución de problemas desde dominios discretos. La categoría central la conforman los subprocesos: transformar, formalizar, generalizar y probar variacionalmente.

Transformación variacional: Formas de sustituir y combinar números enteros que cumplan una relación apropiada y hagan verdadera la ec- uación diofántica $a x+b y=c$ como proceso.

Formalización variacional: Formas de asignar, formular, representar, verificar, organizar y reorganizar como manera de externalizar las operaciones del pensamiento variacional cuando opera sobre la resolución de problemas.

Generalización variacional: Formas de relacionar, investigar (patrones, reglas), extender condiciones y estrategias en la resolución de problemas en una variedad de contextos. 
Prueba variacional: Formas de argumentos, razonamientos, ejemplos, contraejemplos y explicación para convencer de la verdad acerca de los patrones, condiciones, propiedades, relaciones y estrategias en el planteo y resolución de problemas.

\section{Hallazgos y discusión}

Tradicionalmente los profesores de matemáticas esperan que los estudiantes descubran relaciones, generalicen el conocimiento y resuelvan problemas, tal y como lo haría un matemático o un profesor experto. Si de la demostración se trata, el asunto es aún más exigente.

A continuación, se interpretan, describen y dan sentido a las categorías, subcategorías y acciones desde la variación y el cambio que emergen de los datos manifestadas de forma escrita o verbal en el transcurso del proceso cuando los participantes resolvieron problemas desde la variación y el cambio. En la Figura 8, se pueden observar.

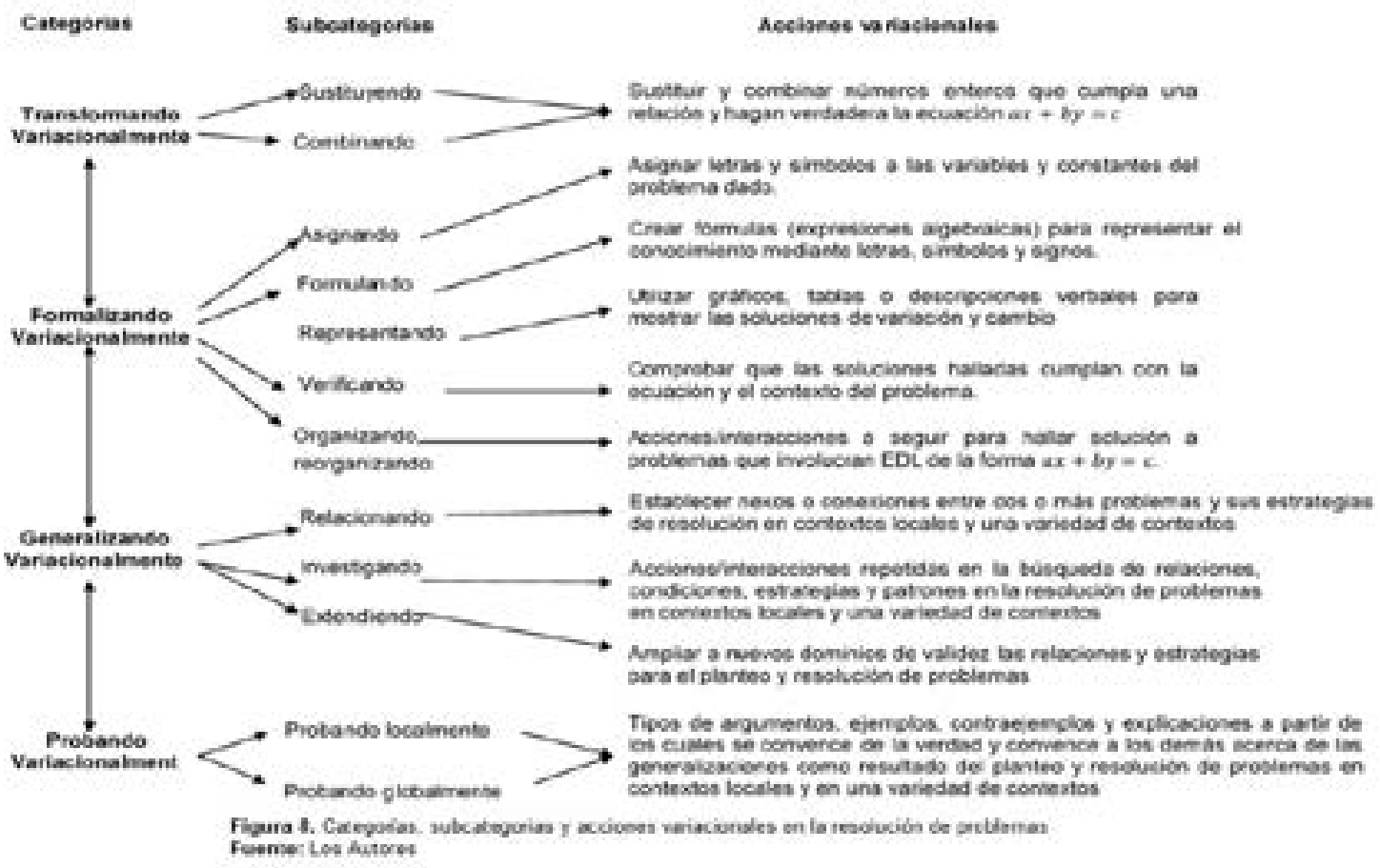

Figura 8. Categorías, subcategorías y acciones variacionales en la resolución de problemas

Fuente: Autores

Se destacan las acciones de los estudiantes cuando utilizan números enteros para reemplazar y combinar las constantes a y $b$, y/o las variables $x$ e $y$, analizan y encuentran reglas, patrones $y$ fórmulas para determinar un número entero en función de los números $a$ y $b$ de acuerdo al tipo de soluciones exigidas a la ecuación (Ver Figura $6)$. El participante también, a partir de sustituciones y combinaciones encuentra relaciones y patrones acerca de cómo cambian los valores de $x$ e $y$ de forma independiente y simultáneamente.

Sobresale también la manera de utilizar letras, signos y símbolos para asignar y formular expresiones algebraicas para representar relaciones, condiciones y patrones como resultado de sustituir y combinar números enteros en la ecuación $a x+b y=c$ de acuerdo a las condiciones del problema, coincidiendo con el 
proceso cognitivo propuesto por Mayer (2010) en la representación del problema, la primera fase de Polya (1945) y la fase de ataque que proponen Mason, Burton y Stacey (2010), (Ver Figuras 4, Figura 6).

Además, las anteriores acciones coinciden y están inmersas en las fases de entrada y ataque propuestas por Mason, Burton y Stacey (2010) quienes afirman que notar ciertas características subyacentes en ejemplos particulares, e ignorar otras, son formas de generalizar. Esto conduce a buscar, si esta generalización es verdadera (conjetura), por qué y dónde es verdadera.

Es notable la manera en que elaboran nexos y construyen relaciones entre problemas actuales y problemas anteriores y sus estrategias de resolución para resolver problemas similares en contextos locales y una variedad de contextos. La manera de organizar y reorganizar acciones e interacciones para investigar relaciones, patrones, condiciones y representarlas mediante fórmulas o expresiones algebraicas se relaciona con generalizar estrategias desde contextos locales a una variedad de contextos (Ver Figuras 4 y Figura 6).

Estas formas de investigar relaciones, organizarlas y reorganizarlas en acciones e interacciones ampliando dominios de validez coincide con las fases de ataque y revisión en los procesos de particularización y generalización de Mason, Burton y Stacey (2010). Estas acciones muestran y coinciden con Burton (1984) y Falk de Losada (1994) en lo que respecta a la importancia de las relaciones cuando se piensa matemáticamente, así como las diferentes formas de establecer y construir estas relaciones

Se hace notar además la manera cómo utilizan ejemplos, contraejemplos y explicaciones como argumentos para convencerse a sí mismo y convencer a los demás, como forma de probar en contextos locales y una variedad de contextos, coincidiendo con Mason, Burton y Stacey (2010) y Harel (2008a; 2008b; 2010) como forma de prueba para convencer, aunque estas acciones no gozan aún de reconocimiento por la comunidad de educación matemática.

\section{CONCLUSIONES}

Para responder la pregunta científica acerca de, ¿Cómo es la naturaleza de la resolución de problemas que involucran ecuaciones lineales diofánticas de la forma $a x+b y=c$ manifestado por profesores de matemáticas en formación?, se diseñó y ejecutó un plan que tuvo como estrategia tres momentos de intervención con los participantes y tres ciclos de codificación y análisis de datos. Los tiempos de intervención y ciclos de codificación, aunque parecen lineales, no lo son. Se interrelacionan y traslapan entre sí de manera permanente. El método de comparación constante, característico de la teoría fundamentada permitió estas interacciones.

La forma de ir comparando y analizando constantemente los datos posibilitó ir de códigos o conceptos iniciales a categorías tentativas desde los datos. Este método paralelo a los procesos de codificación abierta, axial y selectiva condujeron y permitió ir desde los códigos in vivo o resultados manifestados por los participantes, a la teoría formal como resultado del muestreo y saturación teórica.

Finalmente, y como resultado de la investigación, la resolución de problemas desde este contexto se pudo caracterizar como: un flujo permanente de acciones e interacciones variacionales entre los procesos de transformar, formalizar, generalizar y probar, junto a sus subprocesos correspondientes, cuando los participantes solucionan problemas que involucran ecuaciones lineales diofánticas de la forma $a x+b y=c$.

Haciendo una analogía con los trabajos de Polya (1945), Mayer (2010), Schoenfeld (2016), Mason, Burton y Stacey (2010), se caracterizó la resolución de problemas como las 
operaciones de pensamiento variacionales de transformar, formalizar, generalizar y probar con sus respectivas sub operaciones y las acciones variacionales como estrategias de solución, desde la variación y el cambio en dominios discretos.

Además, las investigaciones en educación matemática para intentar construir teoría desde los datos y no desde los matemáticos o profesores expertos han sido poco abordadas. De allí que utilizar la teoría fundamentada, como estrategia para construir teoría aporta a la manera de investigar en educación matemática.

En lo que respecta a la credibilidad de los resultados, los autores consideran que son fiables puesto que provienen de los resultados escritos y verbales de los participantes cuando se enfrentan a este tipo de problemas desde la variación y el cambio. Por otro lado, la manera como fue conceptualizada la resolución de problemas, no es única; posiblemente otro investigador, desde otra mirada puede conceptualizar de forma diferente este proceso.

Desde el punto de vista de reproductividad en otros escenarios, es posible que no se llegue a los mismos resultados, pero las actividades didácticas, sí pueden ser adaptadas y ponerse a prueba. Lo que sí parece ser claro, es que el trabajo realizado desde la teoría fundamentada permitió hacer esta caracterización y deja abierta la posibilidad para seguir investigando procesos desde la variación y el cambio, en otros contextos y dominios.

\section{REFERENCIAS}

Burton, L. (1984). Mathematical Thinking: The Struggle for Meaning. Journal for Research in Mathematics. Education, 15(1), 35-49. doi:10.2307/748986

Charmaz, K. (2006). Constructing grounded theory: A practical guide through qualitative analysis. London: Sage Publications.

Charmaz, K. (2014). Constructing grounded theory ( 2 ed.). Thousand Oaks, CA: Sage.

Corbin, J., \& Strauss, A. (1990). Basics of qualitative research. Sage publications.

Corbin, J., \& Strauss, A. (2008). Basics of qualitative research:Techniques and procedures for developing grounded theory ( 3 ed.). Thousand Oaks, CA, USA: SAGE Publications.

Corbin, J., \& Strauss, A. (2017). Conceptos básicos de la investigación cualitativa: técnicas y procedimientos para desarrollar la teoría fundamentada (4 ed.). Thousand Oaks, California, United States of America: SAGE Publications.

Dreyfus, T. (2002). Advanced mathematical thinking processes. In D. Tall (Ed.), Advanced mathematical thinking (Vol. 11, pp. 25-41). Dordrecht: Springer.

Falk de Losada, M. (1994). Enseñanzas acerca de la naturaleza y el desarrollo del pensamiento matemático extraídas de la historia del álgebra. Boletín de Matemáticas, 1(1), 35-59.

Gagne, R. (1965). The Conditions of Learning. Holt. Rinehart and Winston.

Glaser, B. (1978). Theoretical sensitivity: Advances in the methodology of grounded theory. Mill Valley, CA: Sociology Press.

Glaser, B., \& Strauss, A. (2017). Discovery of grounded theory: Strategies for qualitative research. New York, USA: Routledge.

Glasser, B., \& Strauss, A. (1967). The development of grounded theory. Chicago: IL: Alden. 
Harel, G. (2008a). DNR Perspective on Mathematics Curriculum and Instruction: Focuson Proving,Part I. ZDM-The International Journal on Mathematics Education, 47, 487-500.

Harel, G. (2008b). DNR Perspective on Mathematics Curriculum and Instruction, Part II. ZDM-The International Journal on Mathematics Education.

Harel, G. (2010). DNR-based instruction in mathematics as a conceptual framework. In Theories of mathematics education, 343-367.

Kupisiewicz, C. (1964). O efektívnosti problémového vyučovania: výskum vyučovacích metód matematicko-prírodovedných predmetov.

Mason, J., Burton, L., \& Stacey, K. (2010). Thinking Mathematically (2 ed.). Harlow, UK: Pearson Education Limited.

Mayer, R. (2010). Problem Solving and Reasoning. International Encyclopedia of Education, 273-278. doi:10.1016/B978-008-044894-7.00487-5

Polya, G. (1945). How To Solve It. Princeton: Princeton University Press.

Polya, G. (1981). Mathematical Discovery. New York: Wiley.

Schoenfeld, A. H. (2016). Learning to think mathematically: Problem solving, metacognition, and sense making in mathematics (Reprint). Journal of Education, 196(2), 138. doi:10.1177/002205741619600202

Stanic, G., \& Kilpatrick, J. (1989). Historical perspectives on problem solving in the mathematics curriculum. The teaching and assessing of mathematical problem solving, 3, 1-22. 